\title{
Topical Application of ASN008, a Permanently Charged Sodium Channel Blocker, Shows Robust Efficacy, a Rapid Onset, and Long Duration of Action in a Mouse Model of Pruritus
}

\author{
Roshni Ramachandran, Scott K. Thompson, Shelle Malkmus, Tsutomu Mieda, \\ Jun-Hsiang Lin, Sandeep Gupta, and (D)Tony L. Yaksh \\ Department of Anesthesiology, University of California, San Diego, California (R.R., S.M., T.M., T.L.Y.) and Asana BioSciences, \\ Lawrenceville, New Jersey (S.K.T., J.-H.L., S.G.)
}

Received January 23, 2020; accepted June 22, 2020

\begin{abstract}
The sensation of pruritus, or itch, is associated with a variety of skin and medical disorders. Itch is transmitted through afferent C-fibers, and sodium channels play a key role in the transmission process. Local anesthetics, which block sodium channels, are used topically to treat itch but generally have a short duration of action and are not selective for afferent nerves underlying the itch sensation. Accordingly, there is a substantial unmet need for safe, efficacious, long-acting treatments for chronic pruritus, including nonhistaminergic itch. We investigated the doseresponse, time to onset, and duration of action of ASN008 topical gel, which targets small afferent sodium channels, in a murine model of pruritus in which scratching behavior is induced by intradermal injection of chloroquine into the nape of the neck of C57BL/6 mice. Topical application of ASN008 gel resulted in a concentration-dependent reduction of scratching behavior. Onset of action was $\leq 1$ hour, and duration of scratching inhibition was 15-24 hours. In a further study involving once-daily application for 5 days with chloroquine challenge on day 5 , treatment with ASN008 gel again
\end{abstract}

resulted in a concentration-dependent reduction of chloroquineinduced scratching, even when the gel was removed 3 hours after each daily application. In conclusion, topical ASN008 gel produces a dose-dependent reduction of scratching in a mouse model of pruritus, with a rapid onset and long duration of action, and may prove to be an effective, oncedaily treatment of a variety of pruritic conditions in humans, including nonhistaminergic itch.

\section{SIGNIFICANCE STATEMENT}

ASN008 gel produces a dose-dependent reduction of scratching in a mouse model of pruritus, with a rapid onset and long duration of action, and may prove to be an effective, once- or twice-daily treatment for a variety of pruritic conditions in humans. ASN008 gel has demonstrated good safety and tolerability in healthy volunteers and is currently under investigation in a phase $1 \mathrm{~b}$ clinical study to evaluate safety, tolerability, pharmacokinetics, and preliminary antipruritic efficacy in atopic dermatitis patients (ClinicalTrials.gov ID: NCT03798561).

\section{Introduction}

Itch is an unpleasant sensation that provokes the desire to scratch as a primary response to clear the pruritogen and to terminate the itch sensation. Pruriceptive itch is most commonly associated with a primary skin disorder, as seen in atopic dermatitis, and originates after the activation of peripheral sensory nerve terminals associated with allergic reactions either induced by insect bites or when pruritogens come in contact with the skin. Chronic itch is a debilitating and dominating symptom that accompanies several disorders including skin conditions, such as atopic dermatitis, psoriasis, xerosis, and mastocytosis.

These studies were performed at the University of California, Department of Anesthesiology, as a Laboratory Service agreement by the University of California, San Diego, with Asana Biosciences in the laboratory of T.L.Y.; S.K.T., S.G., and J.-H.L. are employees of Asana Bioscience.

https://doi.org/10.1124/jpet.120.265074.
Pruritus in the absence of primary dermatologic etiology may also be a manifestation of underlying systemic diseases, as in cases of renal and liver failure (Balaskas et al., 1993; Chia et al., 1998; Yosipovitch et al., 2003) or neurologic disorders such as diabetic neuropathy and shingles (Oaklander et al., 2003; Ikoma et al., 2006). The transmission of the pruriceptive itch is through the primary afferent nerve fibers, primarily the slow conducting unmyelinated C-fibers and some thinly myelinated A-delta fibers (Schmelz et al., 1997, 2003) with free nerve endings located in the epidermis that act as sensors in detecting and transmitting pruriceptive signals to the higher brain centers.

Histamine is the best studied pruritogen in mediating itch transduction, and pruritus mechanisms are often classified as histamine-dependent or histamine-independent based on the responsiveness of the itch sensitive neurons to histamine. Histamine released from mast cells has been shown to activate

ABBREVIATIONS: $\mathrm{C}_{\mathrm{Ave}}$, average plasma concentration; CNS, central nervous system; CQ, chloroquine; Mrgpr, mas-related G protein-coupled receptor; TRPA1, transient receptor potential cation channel, subfamily A, member 1; TRPV1, transient receptor potential cation channel, subfamily $\mathrm{V}$, member 1. 
a subset of neurons expressing receptors for transient receptor potential cation channel, subfamily V, member 1 (TRPV1), evidenced by TRPV1 antagonism in histamine-evoked activation of dorsal root ganglion neurons (Shim et al., 2007) and reduced histamine-evoked scratching behavior (Imamachi et al., 2009). However, other pruritogens have emerged in recent years that induce itch via histamine-independent pathways. Chloroquine, an antimalarial drug, causes intolerable itch sensations via such histamine-independent neural pathways (Aghahowa et al., 2010) that use mas-related G protein-coupled receptor (Mrgpr)-initiated signaling. Mrgprs have recently emerged as a novel class of receptors in histamine-independent itch pathways, and MrprA3 is the receptor for chloroquine. In contrast to histamine, chloroquine utilizes transient receptor potential cation channel, subfamily A, member 1 (TRPA1) as a key transduction channel downstream to the MrgprA3 receptor (Liu et al., 2009; Wilson et al., 2011). TRPA1 is highly expressed in TRPV1-positive neurons and is activated downstream to several G protein-coupled receptors that are essential in evoking itch sensations in response to several pruritogens, suggesting TRPA1 as an essential channel in evoking itch (Wilson et al., 2011; Bautista et al., 2014).

Topical local anesthetics are commonly used to treat itch. Conventional local anesthetics are hydrophobic in nature and impair the nociceptive transmission by blocking voltage-gated sodium channels from inside of the cell (Hille, 1977). However, along with short duration of action, these regional anesthetic agents do not specifically target sensory neurons, as they block excitability of all afferent neurons that express sodium channels and hence interfere with the function of autonomic and motor neurons. This highlights a continuing need for new long-lasting and targeted local therapeutic modalities. ASN008 is a permanently charged sodium channel blocker that has been formulated in a polymer-based gel. The structure of ASN008 is provided in Figure 1. Due to its positive charge, ASN008 cannot passively gain access to the interior of the cell to block the sodium channel, but it does pass through activated (open) TRPV1 or TRPA1 channels, which are activated during itch and pain conditions. Selective expression of these transient receptor potential channels on $\mathrm{C}$ - and $\mathrm{A} \delta$-fibers and not on motor neurons allows for nerve-type selective blockade of sodium channels by ASN008. Based on this mechanism, this drug may have a broad application in difficult-to-treat chronic pruritic diseases such as atopic dermatitis.

\section{Materials and Methods}

Animals. Adult male C57BL/6 mice, 25-30 g (Harlan Sprague Dawley Inc., Indianapolis, IN), were housed in the vivarium for

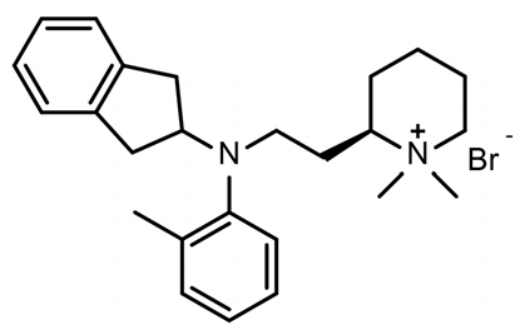

Fig. 1. Chemical structure of ASN008. a minimum of 2 days before use, maintained on a 12-hour day-night cycle and given free access to food and water. All studies were conducted in Association for Assessment and Accreditation of Laboratory Animal Care-approved facilities at the University of California, San Diego, and in accordance with protocols and Standard Operating Procedures approved by the Institutional Animal Care and Use Committee of the University of California, San Diego, and in compliance with the US Department of Agriculture Animal Welfare Act (US Department of Agriculture, Title 9, Code of Federal Regulations, Part 3, Federal Register, February 15, 1991). Animals were housed in the University of California, San Diego, Clinical Teaching Facility Vivarium in a room with temperature maintained in the range of $65-82^{\circ} \mathrm{F}$ and relative humidity in the range of $30 \%-70 \%$. The room was illuminated with fluorescent lighting on a daily 12-hour light/dark cycle. Animals were randomly separated into cages containing approved bedding material. Animals were checked a minimum of once daily, and cages were cleaned a minimum of once weekly. Animals were randomly assigned to dosing groups based on date of receipt at the testing facility.

Drugs and Test Articles. Drugs employed were chloroquine ( $4 \mathrm{mg} / \mathrm{ml}$ ). Chloroquine solutions were prepared from stock and were then serially diluted to the final concentration in $0.9 \%$ saline. All solutions were stored at $4^{\circ} \mathrm{C}$ and brought to room temperature prior to use. ASN008 gel formulations containing $0.3 \%, 1 \%, 3 \%$, and $5 \%$ ASN008, corresponding gel vehicle, lidocaine ointment, and corresponding ointment vehicle, manufactured by Amneal Pharmaceuticals, NJ, were supplied by Asana BioSciences. The 0.1\% ASN008 gel formulation was prepared at the University of California, San Diego, by mixing one part $0.3 \%$ ASN008 gel formulation with two parts vehicle gel.

Drug and Test Article Delivery. Mice were anesthetized (2.5\% isoflurane, with $80 \%$ oxygen and $20 \%$ room air) and were shaven on the dorsolateral aspect of the neck and upper shoulder. ASN008 gel $(0.1 \%, 0.3 \%, 3 \%$, and $5 \%)$ and gel vehicle were applied topically onto the shaved region. Intradermal injections of a solution of chloroquine in $0.9 \%$ saline $(50 \mu \mathrm{l})$ were then administered at different time points to perform the behavioral studies.

Behavioral Assessment. During testing, animals were placed in plexiglass cylindrical chambers, and a detection band was placed around the hind paw ipsilateral to the shaven area. To initiate scratching behavior, intradermal injection of a solution of chloroquine in $0.9 \%$ saline $(50 \mu \mathrm{l})$ was administered in the middle of the shaven area of skin using a $29 \mathrm{G}$ needle. Scratching behavior was recorded over a period of 40 minutes using a paw motion detector. The dose of chloroquine (CQ) and the period of scratches recorded are based on the validated model of CQ-induced scratching over time (Green et al., 2006; Marino et al., 2012; Ramachandran et al., 2018).

Paw Motion Detection for Measuring Itch Responses. The paw motion detection detects the movement of a $0.1-\mathrm{g}$, nonferrous metal band placed around one hind paw of the mouse (Yaksh et al., 2001). The testing apparatus consists of $8.5 \mathrm{~cm}$ diameter, $22.5 \mathrm{~cm}$ tall cylindrical chambers. Under each cylinder is a pair of circular concentric electromagnetic coils that serve as antennae for transmission and reception. The outer coil diameter is $12 \mathrm{~cm}$. The transmitter coil assembly is constructed to emit a $5-8 \mathrm{~mW}, 6-8$ $\mathrm{kHz}$, sinusoidal electromagnetic field (Blue Max 800 Precision scan search coil; White's Electronics, Inc., Sweet Home, OR). The detection principal is that eddy currents created by the movements of the ferrous and nonferrous metals perturb the electromagnetic field. Such perturbations produce an output waveform and are subsequently detected (Yaksh et al., 2001).

Plasma Sample Collection and Analysis. Plasma samples were collected immediately after completion of scratching behavior recording in the two concentration-response studies. For the singledose concentration-response study, plasma samples were collected from the groups treated with the 3\% and 5\% ASN008 gels. For the 
5-day repeat-dose concentration-response study, plasma samples were collected for all groups treated with ASN008 gels $(0.1 \%, 0.3 \%$, $1 \%$ and $3 \%$ ). Samples were analyzed using liquid chromatography with tandem mass spectrometry.

Statistical Analysis. The data for each variable was put in tabular form (i.e., Excel worksheet). Summary statistics were computed and include group means, S.D.s, and numbers of animals per group. Statistical analysis was performed using GraphPad Prism 6, version 6.0c (GraphPad Software, San Diego, CA). Analysis of the automated scratching data sets generated in pooled control groups and in pooled chloroquine treated groups did not reject the hypothesis at the $P<0.05$ level of either normality (D'Agostino and Pearson omnibus normality test) or homogeneous variances (Bartlett's test). Accordingly, for comparison of chloroquine-induced scratching, results were compared using a one-way ANOVA across doses or time with Bonferroni post hoc tests to compare groups at similar doses or times. For all post hoc comparisons, multiplicity adjusted $P$ values were calculated. In each case, Bonferroni post hoc tests (e.g., $t$ tests with Bonferroni corrections) were undertaken and

A

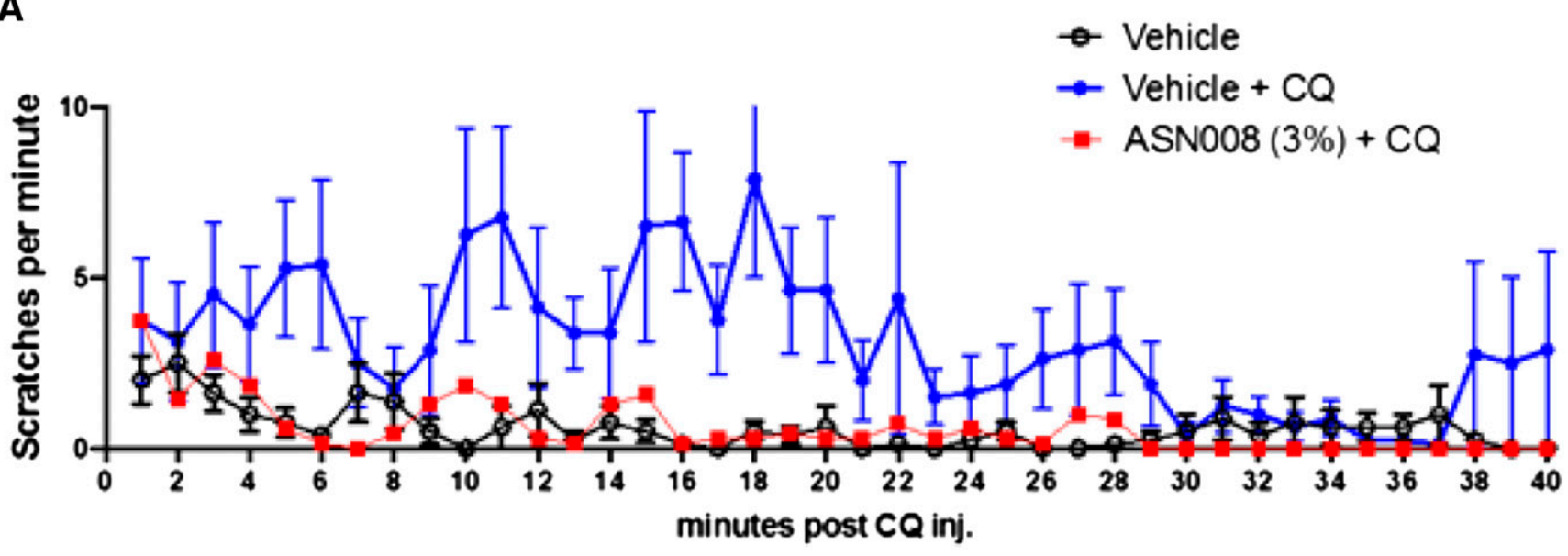

B

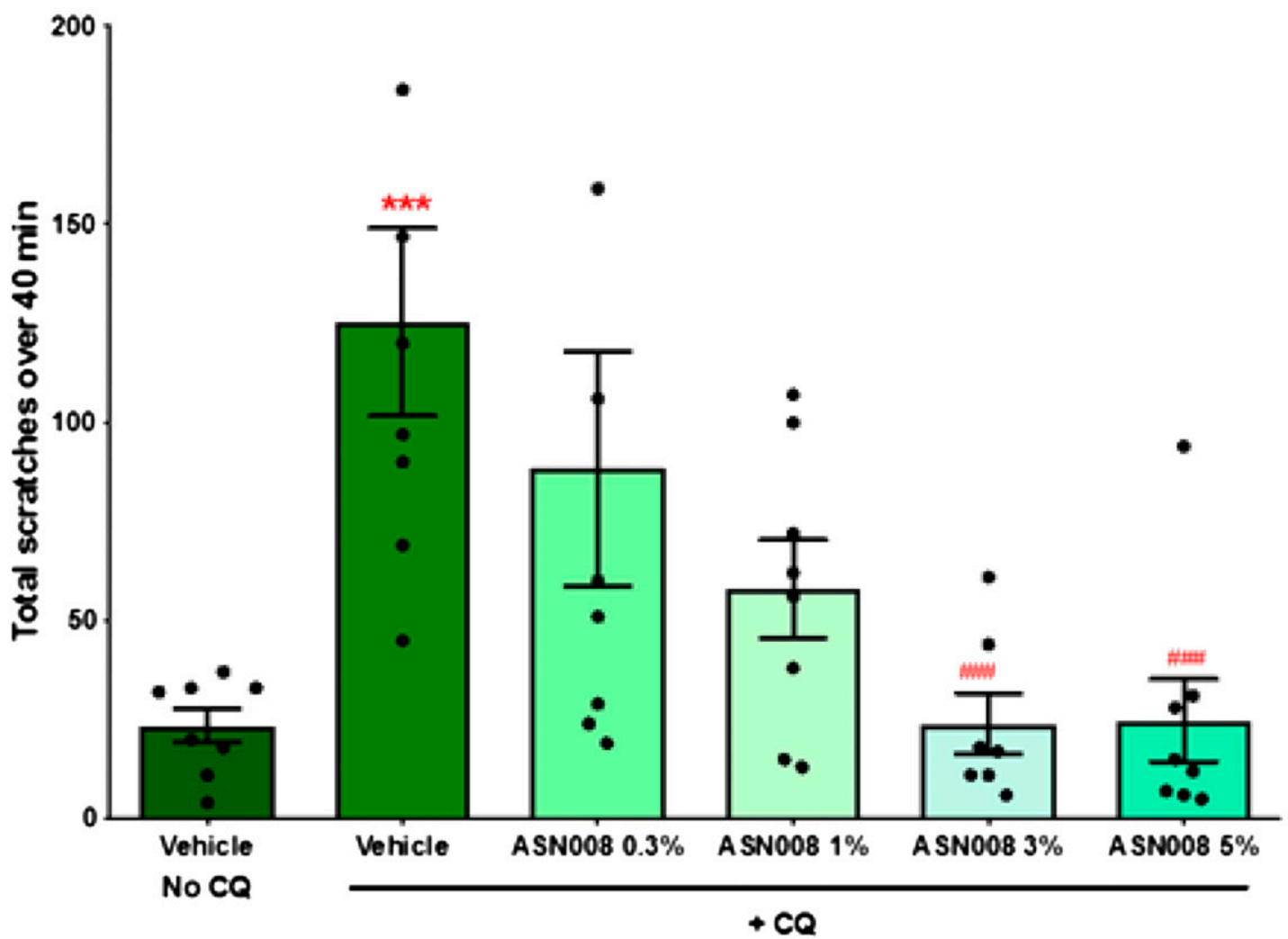

Fig. 2. Concentration-response. (A) Scratches per minute over a period of 40 minutes. (B) Bar graph with scatter dot plot shows the total number of scratches over a 40-minute period with or without intradermal CQ injection 6 hours after the application of gel vehicle and different gel formulations with varying concentrations of ASN008 gel $(0.3 \%, 1 \%, 3 \%$, and 5\%). Animals pretreated with vehicle showed a significant increase in the number of scratches after intradermal chloroquine. The animals pretreated with 3\% and 5\% ASN008 gel showed significantly reduced CQ-induced scratching as compared with animals pretreated with gel vehicle. Plots indicate means \pm S.E.M. for cumulative scratches. $* * * P<0.001$ vs. vehicle with no CQ;

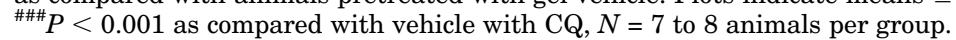


are presented in the graphics and figure legends for values between $P<0.01$ and $P<0.0001$.

\section{Results}

\section{Concentration-Dependent Effect of Single Topical Application of ASN008 Gel on Intradermal Chloroquine-Induced Scratching}

Chloroquine-Induced Scratching. In the absence of the chloroquine stimulus, only occasional brief episodes of grooming involving the hind paw were noted and were counted. After intradermal injection of $\mathrm{CQ}$, the animal displayed a brisk scratching of the injected nape skin with the ipsilateral hind paw. This scratching behavior was characterized by long durations of high frequency bouts of paw movement recorded by the machine detection algorithm as a series of scratch counts (Marino et al., 2012). Scratches were recorded for 40 minutes. CQ injected unilaterally induced ipsilateral scratching behavior. The total number of scratches in the 40minute period showed a significant increase (5-fold) after intradermal injection of CQ (125.4 \pm 23.68 scratches) compared with the gel vehicle group ( $23.50 \pm 4.25$ scratches $)$, and scratching persisted unabated for intervals in excess of 4060 minutes (Fig. 2A).

ASN008 Concentration-Response. To determine the minimum effective concentration of a single application of ASN008, a concentration-response study was performed in which the mice were pretreated with different concentrations
A

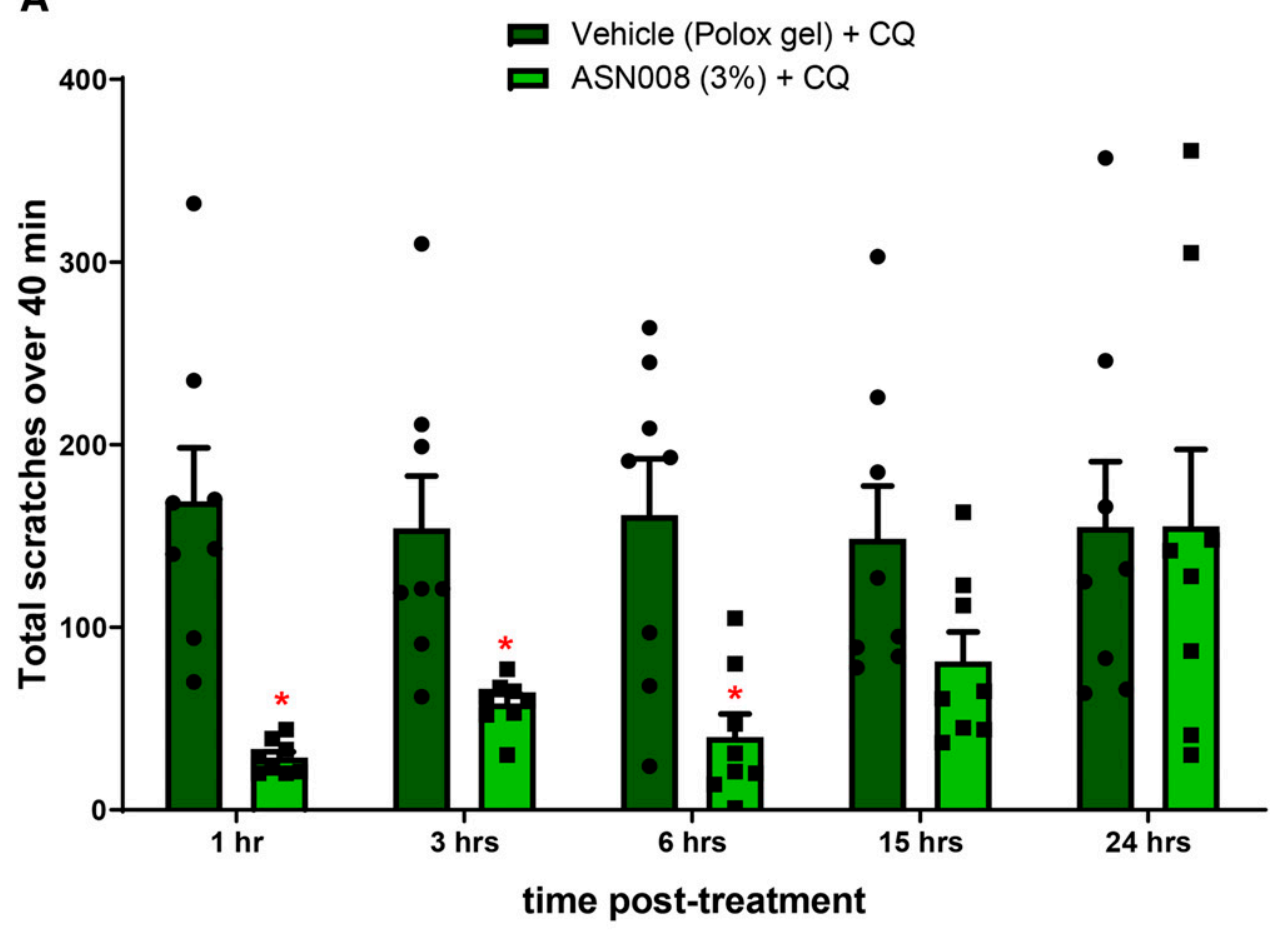

B
Fig. 3. Bar graph with scatter dot plot depicts total number of scratches over a 40-minute period. (A) Bar graph shows onset and duration of antipruritic action of 3\% ASN008 gel on CQ-induced scratching. The onset of antipruritic action of $3 \%$ ASN008 gel was $\leq 1$ hour (earliest time point measured), and the duration of action was between 15 and 24 hours as compared with the vehicle control at respective time points. Plots indicate means \pm S.E.M. for cumulative scratches. $* P<0.05$ vs. vehicle at respective time points. (B) Bar graph shows antipruritic action of 5\% lidocaine ointment on CQinduced scratching. Onset of antipruritic action of lidocaine was $\leq 1$ hour, and the effect completely reversed by 6 hours. $* P<0.05$ vs. vehicle at respective time points. $N=8$ animals per group.

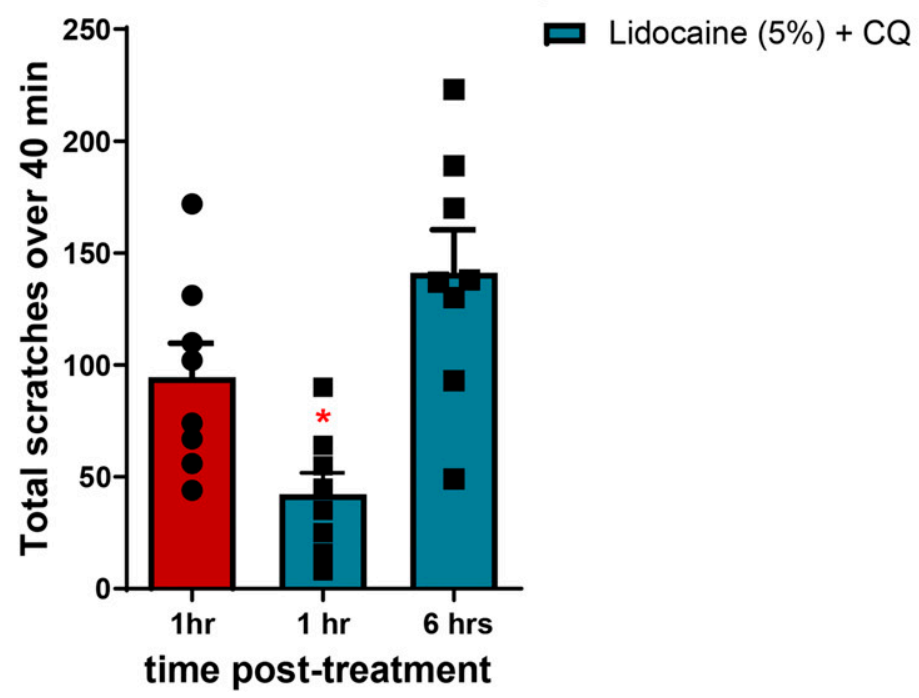


of ASN008 [0\% (vehicle), $0.3 \%, 1 \%, 3 \%$, and $5 \%$ ] prior to CQ injection. ASN008 gel formulations $(50 \mu \mathrm{l})$ were applied topically to the nape of the neck 6 hours prior to intradermal $\mathrm{CQ}(4 \mathrm{mg} / \mathrm{ml}, 50 \mu \mathrm{l})$ injections at the same site. A significant reduction in CQ-induced scratching behavior was observed after topical application of $3 \%(24.0 \pm 7.74)$ (Fig. 2) and $5 \%(24.75 \pm 1.47)$ (Fig. 2B) ASN008 gels. Reductions in scratching behavior were observed after treatment with the $0.3 \%$ and $1 \%$ ASN008 gels, but they were not statistically significant compared with treatment with gel vehicle (Fig. 2B). Mice in these groups did not per se display scratching at the site after test article or vehicle application alone. Motor function (measured by placing and stepping) and pinna and corneal reflexes were normal after the application of the drug. No weaknesses were observed in any of the study animals.

\section{Time of Onset and Duration of Action of 3\% ASN008 Gel on Chloroquine-Induced Scratching}

Since ASN008 (3\%) was the most effective concentration that showed a significant inhibition on $\mathrm{CQ}$-induced scratching (Fig. 2A), we examined the time course effect of the $3 \%$ ASN008 gel in the CQ-induced pruritus model. Mice were pretreated with $3 \%$ ASN008 gel at various time points $(1,3,6$, 15 , and 24 hours) prior to the injection of CQ ( $50 \mu \mathrm{l}, 4 \mathrm{mg} / \mathrm{ml})$. Another group of mice received the vehicle pretreatment at similar time points. The scratching behavior was recorded over the period of 40 minutes. The vehicle group showed a significant increase in the number of scratches after intradermal CQ injection at all time points $(\sim 169 \pm 29.23)$. The animals pretreated with $3 \%$ ASN008 gel showed significant inhibition of CQ-induced scratches, starting at 1 hour (28.62 \pm 3.27 ) and lasting up to 6 hours (39.87 \pm 12.61$)$, with a partial reversal observed at 15 hours $(81.25 \pm 16.20)$. By 24 hours, complete return of $\mathrm{CQ}$-induced scratching was observed $(155.25 \pm 42.07)$ (Fig. 3A). The effect of $5 \%$ lidocaine ointment pretreatment was also studied. The inhibitory effect of $5 \%$ lidocaine ointment on CQ-induced scratching was observed as early as 1 hour (42.25 \pm 9.57$)$, as compared with its vehicle control. However, a complete return of CQ-induced scratching was observed by 6 hours after lidocaine application (141.1 \pm 19.26) (Fig. 3B).

To evaluate the effect of the absorbed drug and its ongoing efficacy after removal of the gel, 3\% ASN008 gel was applied topically to the skin and removed at 3 hours after application by gentle lavage, and the effects on intradermal CQ (4 mg/ml, $50 \mu \mathrm{l}$ )-induced scratching were tested in separate groups at three different time points ( 3,6 , and 15 hours) after test article application. Separate groups without the removal of the drug were also included for reference purposes. The group with the removal of the drug showed similar time-dependent inhibitory effects on CQ-induced scratching as observed in the group without the removal of the drug (Fig. 4).

\section{Effect of the Successive Daily Dosing of Different Concentrations of ASN0008 on Chloroquine-Induced Scratching Behavior}

This study examined the antipruritic effect of successive once-daily dosing of ASN0008 formulations for five consecutive days at $0.1 \%, 0.3 \%, 1 \%$, and $3 \%$ and vehicle on CQ-induced scratching behavior on day 5 . Two other groups were included in this study. One group was examined for repeated application of placebo, and the baseline scratching was recorded on day 5 without intradermal $\mathrm{CQ}$ administration. The other group received topical application of ASN008 (3\%), which was removed with water after 3 hours after the application every day for 5 days. Once-daily application for 5 days with CQ challenge on day 5 , treatment with $1 \%$ ASN008 (53.25 \pm 6.54$)$ or $3 \%$ ASN008 gel $(35.0 \pm 12.61)$, reliably resulted in complete blockade of the chloroquine-induced scratching (Fig. 5), even when the $3 \%$ gel was removed 3 hours after each daily application (data not shown), consistent with the results from a single-application study examining gel removal after 3 hours (Fig. 4).

\section{Plasma Levels of ASN008}

Plasma samples were taken from animals approximately 7 hours after application of the $3 \%$ or $5 \%$ ASN008 gels in the single-dose concentration-response study and approximately 4 hours after application of the $0.1 \%, 0.3 \%, 1 \%$, and $3 \%$ ASN008 gels on day 5 of the 5-day repeat-dose concentration-response study. Data are summarized in Table 1. Plasma levels of ASN008 in animals receiving 3\% or $5 \%$ ASN008 gels in the single application study were found to be low and highly variable [average plasma concentration $\left(\mathrm{C}_{\text {Ave }}\right)=4.4 \mathrm{ng} / \mathrm{ml}, \mathrm{CV}=118 \%$ in the $3 \%$ group $\mathrm{C}_{\text {Ave }}=3.8 \mathrm{ng} / \mathrm{ml}$, $\mathrm{CV}=118 \%$ in the $5 \%$ group]. In the 5 -day repeat-dose concentration-response study, plasma samples taken after application of the $0.1 \%, 0.3 \%, 1 \%$, and $3 \%$ ASN008 gels were found to be low, although higher than in the single application

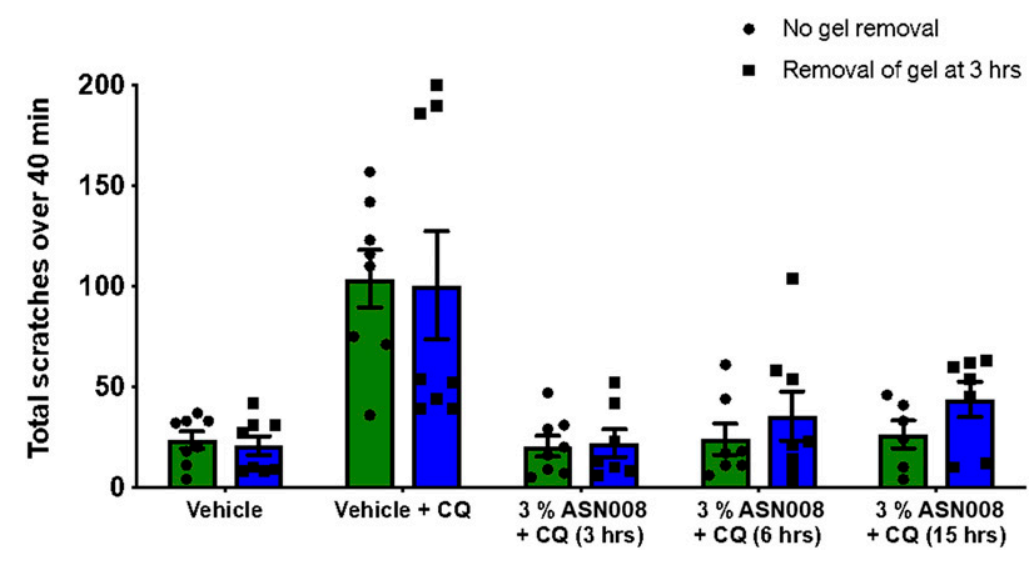

Fig. 4. Bar graph shows total number of scratches in the 40minute period after $\mathrm{CQ}$ injection 3,6, and 15 hours after the application of ASN008 gel (3\%) or gel vehicle alone with or without its removal at 3 hours. Animals pretreated with vehicle gel showed a significant increase in the number of scratches after intradermal chloroquine injection. The animals pretreated with ASN008 gel (3\%) with or without removal showed similar effects. Chloroquine-induced scratching was inhibited in both groups starting at 3 hours and lasted for 15 hours, and this reduction was statistically significant as compared with the group that was pretreated only with the vehicle. Plots indicate means \pm S.E.M. for cumulative scratches. $N=8$ animals per group. 


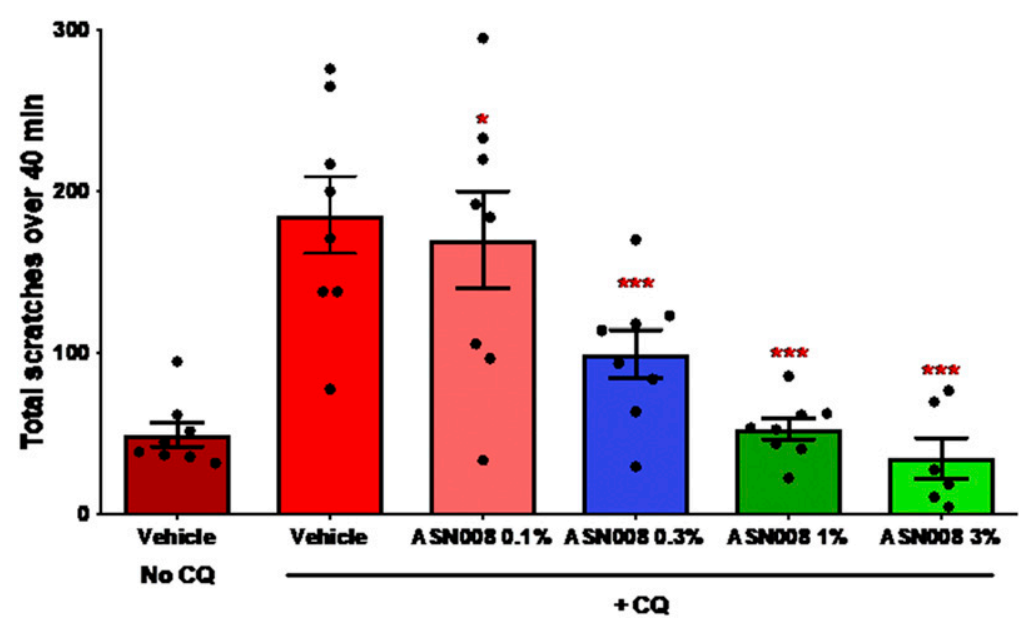

Fig. 5. Bar graph and scatter dot plot represents the total number of scratches after intradermal CQ injection over a 40 minute period on day 5 after once-daily application of ASN008 gel $(0.1 \%, 0.3 \%, 1 \%$, and $3 \%)$ or vehicle gels for 5 days. Successive application of ASN008 for 5 days resulted in significant reduction of chloroquine-induced scratching with ASN008 gel $(0.3 \%)$ and showed a complete blockade with the $1 \%$ and 3\% ASN008 gels as compared with the vehicle. Plots indicate means \pm S.E.M. for cumulative scratches. $* P<0.05$; ***P $P<0.001$ vs. vehicle with $\mathrm{CQ} ; N=7$ to 8 animals per group.

study, moderately variable, and concentration-dependent. In the group treated with the $0.1 \%$ ASN008 gel, plasma levels of ASN008 were below the level of quantitation $(0.5 \mathrm{ng} / \mathrm{ml})$. In the group treated with the $0.3 \%$ gel, four of the animals had plasma levels of ASN008 below the limit of quantitation, whereas in the remaining four animals, the average level was $1.0 \mathrm{ng} / \mathrm{ml}$ with a CV value of $37 \%$. In the groups treated with the $1 \%$ and $3 \%$ ASN008 gels, the average plasma levels of ASN008 were 9.6 and $27 \mathrm{ng} / \mathrm{ml}$, respectively, with CV values of $58 \%$ and $34 \%$, respectively (Fig. 6; Table 1).

\section{Discussion}

The present study demonstrates that intradermal injection of chloroquine results in a robust and highly reliable scratching response. Previous work has shown that this chloroquine effect was not blocked by diphenhydramine, consistent with its histamine-independent effect (Marino et al., 2012). This effect of chloroquine is believed to be mediated by activation of Mrg (Liu et al., 2009; Aghahowa et al., 2010; Ru et al., 2017), which is expressed in TRPV1- and TRPA1-expressing small primary afferent nerves (Hager et al., 2008; Akiyama and Carstens, 2014).

Mechanisms of Therapeutic Actions. Targeting the sodium channels with local regional anesthetics such as lidocaine has been shown to have significant effects upon the sensation of pruritus (Layton and Cotterill, 1991; Allenby et al., 1993; Kopecky et al., 2001; Patel and Yosipovitch, 2010). However, as local anesthetics typically exert their action from an intracellular site, penetration through the cell membrane requires a lipophilic state to penetrate and then a re-protonation in the more acidic intracellular environment, allowing it to it efficaciously block the voltage-gated sodium channel (Hille, 1977; Butterworth and Strichartz,
1990). There are two limiting issues related to the use of this therapeutic approach. First, such uptake targets not only the nociceptive or pruriceptive neurons but also motor and autonomic axons. Second, lipophilicity leads to a more rapid vascular uptake and loss of therapeutic activity. As noted, ASN008 is a permanently charged sodium channel blocker that contains a quaternary amino functionality. Because of its permanent positive charge, ASN008 cannot gain access to the interior of the cell by passively permeating through the cell membrane. However, ASN008 is believed to gain access to the interior of the cell by passage through the large gated pores present in TRPA1 and TRPV1 channels. These channels, when activated, allow for the permissive movement of the quaternary molecule into the intracellular compartment where it then has access to the sodium channel. Importantly, it is the charged (quaternary) form of the molecule that is the active entity in such channel blocks. This restricted access of the quaternary form thus endows the approach with an inherent specificity resulting in a block that is limited to membranes with such large access pores and the presence of conditions, leading such pores to be activated (opened).

Effect of ASN008, a Permanently Charged Quaternary Amine Sodium Channel Blocker. The present study investigated the concentration-response, duration of action, and continuous application of the quaternary amine sodium channel blocker ASN008 on chloroquine-induced scratching behavior. Topical application of ASN008 gel resulted in a concentration-dependent reduction of scratching in a mouse model of pruritus. A single application of 3\% ASN008 gel significantly reduced chloroquine-induced scratching. A rapid onset and long duration of suppression of this robust scratching behavior was also observed compared with the conventional local anesthetics such as lidocaine, a tertiary amine that does not bear a permanent positive charge and that has a very

TABLE 1

Plasma levels of ASN008 from concentration-response studies

\begin{tabular}{|c|c|c|c|c|c|c|}
\hline \multirow[b]{2}{*}{ ASN008 concentration in gel } & \multicolumn{2}{|c|}{ Single application ${ }^{a}$} & \multicolumn{4}{|c|}{ Once-daily application for 5 days $^{a}$} \\
\hline & $3 \%$ & $5 \%$ & $0.1 \%$ & $0.3 \%$ & $1 \%$ & $3 \%$ \\
\hline ASN008 $C_{\text {Ave }}(\mathrm{ng} / \mathrm{ml})$ & 4.4 & 3.2 & $<0.5$ & $1.0^{b}$ & 9.6 & 27 \\
\hline S.D. (ng/ml) & 5.2 & 3.8 & N/A & 0.39 & 5.5 & 9.2 \\
\hline Coefficient of variation (\%) & 118 & 118 & N/A & 37 & 58 & 34 \\
\hline
\end{tabular}

${ }^{a} N=8$ animals/group.

${ }^{b}$ Plasma level $<0.5 \mathrm{ng} / \mathrm{ml}$ in four of eight animals; $\mathrm{C}_{\mathrm{Ave}}$ calculated from plasma levels in four animals with $\mathrm{C}_{\mathrm{Ave}}$ values above $0.5 \mathrm{ng} / \mathrm{ml}$. 
A

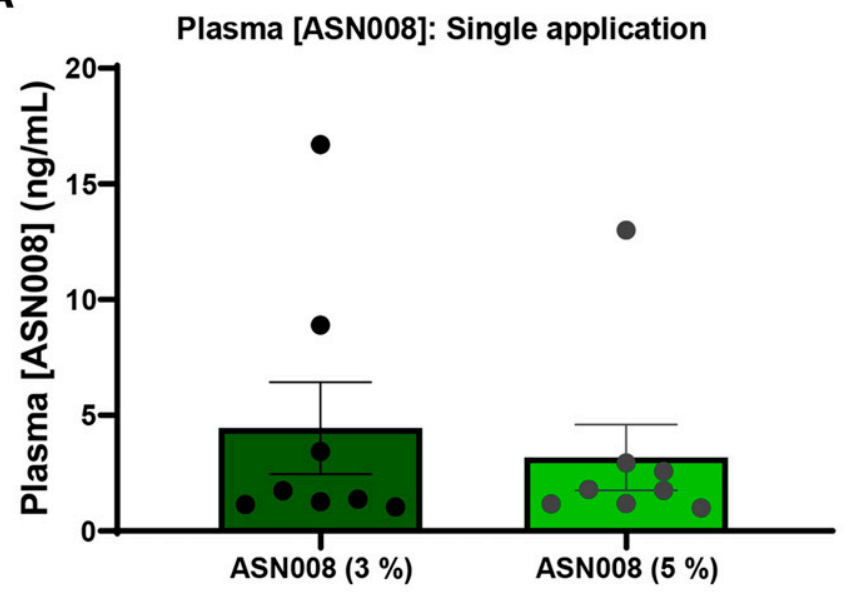

B

Plasma [ASN008]: 5-day-repeated application

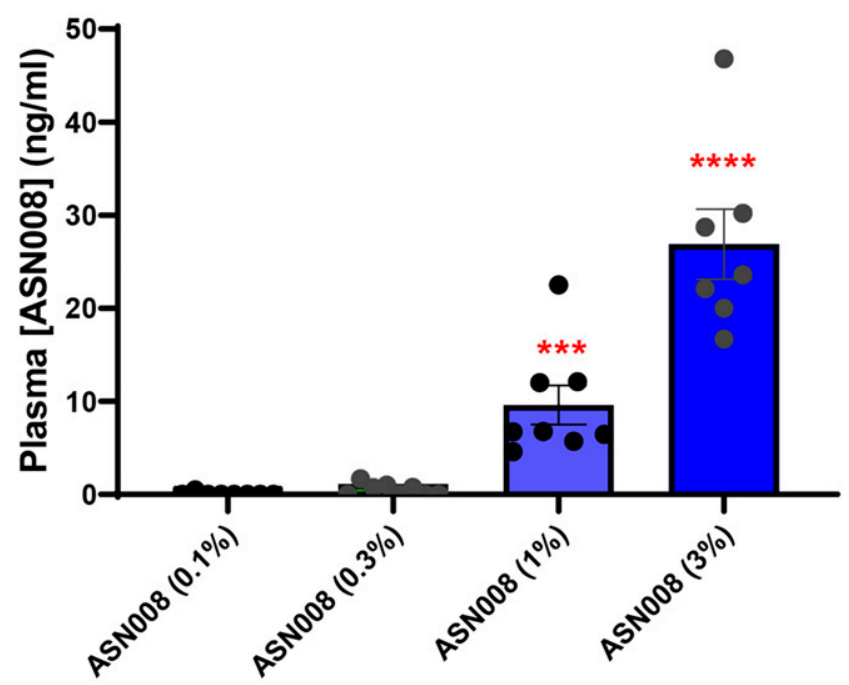

Fig. 6. Bar graph with scatter dot plot represents plasma levels of ASN008 after single application (A) and repeated once-daily application (for 5 days) (B) of ASN008 gel using different concentrations. The plasma levels of ASN008 in the 5-day repeat-dose study were significantly higher in the $1 \%$ and $3 \%$ groups than the $3 \%$ and $5 \%$ groups in the single-dose study. Plots indicate means \pm S.E.M. for plasma ASN008 concentration. $* * * P<0.001 ; * * * * P<0.0001$ vs. ASN008 (0.1\%) and ANS008 (0.3\%); $N=7$ to 8 animals per group.

short duration of action. Repeated application of the ASN008 gel showed no signs of tachyphylaxis, consistent with its anticipated mechanisms of action, blocking of small afferent sodium channels.

Topical application of ASN008 gel resulted in a long duration of action. This duration of effect is considered to reflect the long residence time (e.g., slow clearance) of the quaternary molecule from inside the axon and poor local vascular absorption of the highly polar molecule. Assessment of plasma drug levels revealed a dose-dependent plasma concentration for ASN008, which at the therapeutic concentration of $3 \%$ resulted in a peak plasma concentration of $<30 \mathrm{ng} / \mathrm{ml}$. In contrast to these relatively low plasma concentrations after therapeutic dosing of the charged ASN008 in gel formulation
(3\%), a eutectic mixture of lidocaine $2.5 \%$ applied to the normal mouse skin for management of soft tissue wound pain revealed considerably greater $\mathrm{C}_{\max }$ values of $160 \mathrm{ng} / \mathrm{ml}$ (AlMusawi et al., 2016). Also possibly contributing to the long duration of action is the potential for the skin tissue to act as a depot for drug accumulation. This is supported by the observation that plasma levels of ASN008 in the 5-day repeat-dose study were substantially higher in the $1 \%$ and $3 \%$ groups than the $3 \%$ and $5 \%$ groups in the single-dose study, indicative of drug accumulation.

Preclinical Safety. Based on preclinical dermal toxicological evaluation (acute primary skin irritation study in rabbits, skin sensitization in guinea pigs, and 28-day repeatdose dermal toxicity study in minipigs), topical administration of ASN008 gel in the same formulation at $\leq 3 \%$ was well tolerated without adverse irritation or sensitization findings on the treated skin. Findings in the dermal toxicity study were limited to the skin (erythema, edema, and eschar), were nonadverse, and were fully reversible. In addition, based on the preclinical systemic toxicological and safety pharmacology studies [28-day repeat-dose intravenous toxicity study in rats and single-dose subcutaneous central nervous system (CNS) and respiratory studies in rats], there were no adverse findings in any tissues or on CNS or respiratory systems, including motor function up to the maximum doses administered $(5 \mathrm{mg} / \mathrm{kg}$ per day in the 28-day repeat-dose intravenous study, $10 \mathrm{mg} / \mathrm{kg}$ in the single-dose subcutaneous CNS study, and $8 \mathrm{mg} / \mathrm{kg}$ in the single-dose subcutaneous respiratory study). The battery of assessments conducted in the CNS studies are summarized in Table 2. The only findings in the respiratory study were transient, nonadverse increases in respiratory rate and minute volume and lower tidal volume at 30-40 minutes after administration of ASN008. Of note, systemic exposure levels of ASN008 measured in these studies $(1095 \pm 309 \mathrm{ng} / \mathrm{ml}$ at $5 \mathrm{mg} / \mathrm{kg}$ in the 28 -day repeatdose intravenous study and $1070 \pm 188 \mathrm{ng} / \mathrm{ml}$ at $10 \mathrm{mg} / \mathrm{kg}$ in the single-dose subcutaneous study) were over 100 -fold higher than concentrations measured at the minimum fully efficacious concentration of $1 \%$ ASN008 in the 5-day repeatdose concentration-response study in the mouse pruritus model $(9.6 \pm 5.5 \mathrm{ng} / \mathrm{ml}$, Table 1$)$. Overall, ASN008 gel is well tolerated in dermal toxicology studies at concentrations that are efficacious in the mouse pruritus model, and in systemic toxicology studies at doses leading to systemic concentrations of ASN008 that are more than 100-fold higher than systemic concentrations measured at the minimum fully efficacious concentration in the mouse pruritus model.

\section{Conclusions}

These results with the therapeutic effects of topical ASN008 upon chloroquine-induced scratching in the mouse are of particular interest as, unlike other models of pruritus, molecular studies of CQ-induced pruritus suggest a mechanistic phenotype for the pruritus that accompanies other diseases such as chronic kidney disease, chronic liver disease, skin disorders, and burns (McNeil and Dong, 2014; Ajayi, 2019). The rapid onset of action and long-lasting effect of the topical ASN008 application suggest this to be an inviting strategy for developing a selective and well tolerated therapy for pruritus. 
TABLE 2

Functional observation battery assessments in CNS safety pharmacology studies

\begin{tabular}{|c|c|c|}
\hline Observation category & Assessment & Findings \\
\hline Home cage observations & Posture, convulsions/tremors, biting, eyelid closure, feces consistency & None \\
\hline Handling observations & $\begin{array}{l}\text { Ease of removal from cage, ease of handling animal in hand, lacrimation/ } \\
\text { chromodacryorrhea, salivation, piloerection, fur appearance, palpebral closure, } \\
\text { respiratory rate/character, eye prominence, mucous membranes/eye/skin color, red/ } \\
\text { crusty deposits, muscle tone }\end{array}$ & None \\
\hline Open field observations & $\begin{array}{l}\text { Mobility, rearing, convulsions/tremors, grooming, bizarre/stereotypic behavior, time to } \\
\text { first step (seconds), gait, gait score, arousal, backing, urination/defecation }\end{array}$ & None \\
\hline Sensory observations & $\begin{array}{l}\text { Approach response, touch response, startle response, tail pinch response, pupil } \\
\text { response, eyeblink response, forelimb extension, hindlimb extension, air righting } \\
\text { reflex, olfactory orientation }\end{array}$ & None \\
\hline $\begin{array}{c}\text { Neuromuscular } \\
\text { observations }\end{array}$ & $\begin{array}{l}\text { Hindlimb extensor strength, hindlimb foot splay, grip strength (hind- and forelimb), } \\
\text { rotarod performance }\end{array}$ & None \\
\hline $\begin{array}{l}\text { Physiologic } \\
\text { observations }\end{array}$ & Catalepsy, body temperature, body weight & None \\
\hline
\end{tabular}

\section{Authorship Contributions}

Contributed to research design: Ramachandran, Thompson, Malkmus, Gupta, Yaksh.

Conducted experiments: Ramachandran, Malkmus.

Performed data analysis: Ramachandran, Malkmus, Yaksh.

Contributed to the writing of the manuscript: Ramachandran, Thompson, Malkmus, Lin, Gupta, Yaksh.

\section{References}

Aghahowa SE, Obianwu HO, Isah AO, and Arhewoh IM (2010) Chloroquine-induced pruritus. Indian J Pharm Sci 72:283-289.

Ajayi AAL (2019) Itching, chloroquine, and malaria: a review of recent molecular and neuroscience advances and their contribution to mechanistic understanding and therapeutics of chronic non-histaminergic pruritus. Int J Dermatol 58:880-891.

Akiyama T and Carstens E (2014) Spinal coding of itch and pain, in Itch: Mechanisms and Treatment (Carstens E and Akiyama T eds) pp 319-338, CRC Press/Taylor \& Francis, Boca Raton, FL.

Allenby CF, Johnstone RS, Chatfield S, Pike LC, and Tidy G (1993) PERINAL--a new no-touch spray to relieve the symptoms of pruritus ani. Int $J$ Colorectal Dis 8 184-187.

Al-Musawi A, Matar K, Kombian S, and Andersson L (2016) Blood concentration of prilocaine and lidocaine after the use of topical anesthesia $\left(\right.$ Oraqix $\left.{ }^{\circledR}\right)$ in lacerated wounds. Dent Traumatol 32:502-506.

Balaskas EV, Chu M, Uldall RP, Gupta A, and Oreopoulos DG (1993) Pruritus in continuous ambulatory peritoneal dialysis and hemodialysis patients. Perit Dial Int 13 (Suppl 2):S527-S532.

Bautista DM, Wilson SR, and Hoon MA (2014) Why we scratch an itch: the molecules, cells and circuits of itch. Nat Neurosci 17:175-182.

Butterworth JF IV and Strichartz GR (1990) Molecular mechanisms of local anesthesia: a review. Anesthesiology 72:711-734.

Chia S-C, Bergasa NV, Kleiner DE, Goodman Z, Hoofnagle JH, and Di Bisceglie AM (1998) Pruritus as a presenting symptom of chronic hepatitis C. Dig Dis Sci 43 2177-2183.

Green AD, Young KK, Lehto SG, Smith SB, and Mogil JS (2006) Influence of genotype, dose and sex on pruritogen-induced scratching behavior in the mouse. Pain 124:50-58.

Hager UA, Hein A, Lennerz JK, Zimmermann K, Neuhuber WL, and Reeh PW (2008) Morphological characterization of rat Mas-related G-protein-coupled receptor C and functional analysis of agonists. Neuroscience 151:242-254.

Hille B (1977) Local anesthetics: hydrophilic and hydrophobic pathways for the drugreceptor reaction. J Gen Physiol 69:497-515.

Ikoma A, Steinhoff M, Ständer S, Yosipovitch G, and Schmelz M (2006) The neurobiology of itch. Nat Rev Neurosci 7:535-547.

Imamachi N, Park GH, Lee H, Anderson DJ, Simon MI, Basbaum AI, and Han SK (2009) TRPV1-expressing primary afferents generate behavioral responses to pruritogens via multiple mechanisms. Proc Natl Acad Sci USA 106: $11330-11335$.

Kopecky EA, Jacobson S, Bch MB, Hubley P, Palozzi L, Clarke HM, and Koren G (2001) Safety and pharmacokinetics of EMLA in the treatment of postburn pruritus in pediatric patients: a pilot study. J Burn Care Rehabil 22:235-242.

Layton AM and Cotterill JA (1991) Notalgia paraesthetica--report of three cases and their treatment. Clin Exp Dermatol 16:197-198.

Liu Q, Tang Z, Surdenikova L, Kim S, Patel KN, Kim A, Ru F, Guan Y, Weng HJ, Geng Y, et al. (2009) Sensory neuron-specific GPCR Mrgprs are itch receptors mediating chloroquine-induced pruritus. Cell 139:1353-1365.

Marino M, Huang P, Malkmus S, Robertshaw E, Mac EA, Shatterman Y, and Yaksh TL (2012) Development and validation of an automated system for detection and assessment of scratching in the rodent. $J$ Neurosci Methods 211:1-10.

McNeil B and Dong X (2014) Mrgprs as itch receptors, in Itch: Mechanisms and Treatment: Frontiers in Neuroscience (Carstens E and Akiyama T eds), CRC Press/ Taylor \& Francis, Boca Raton, FL.

Oaklander AL, Bowsher D, Galer B, Haanpää M, and Jensen MP (2003) Herpes zoster itch: preliminary epidemiologic data. J Pain 4:338-343.

Patel T and Yosipovitch G (2010) Therapy of pruritus. Expert Opin Pharmacother 11: 1673-1682.

Ramachandran R, Marino MJ, Paul S, Wang Z, Mascarenhas NL, Pellett S, Johnson EA, DiNardo A, and Yaksh TL (2018) A study and review of effects of Botulinum Toxins on mast cell dependent and independent pruritus. Toxins (Basel) 10:134 Available from: 10.3390/toxins10040134.

Ru F, Sun H, Jurcakova D, Herbstsomer RA, Meixong J, Dong X, and Undem BJ (2017) Mechanisms of pruritogen-induced activation of itch nerves in isolated mouse skin. J Physiol 595:3651-3666.

Schmelz M, Schmidt R, Bickel A, Handwerker HO, and Torebjörk HE (1997) Specific C-receptors for itch in human skin. $J$ Neurosci 17:8003-8008.

Schmelz M, Schmidt R, Weidner C, Hilliges M, Torebjörk HE, and Handwerker HO (2003) Chemical response pattern of different classes of C-nociceptors to pruritogens and algogens. J Neurophysiol 89:2441-2448.

Shim W-S, Tak MH, Lee MH, Kim M, Kim M, Koo JY, Lee CH, Kim M, and Oh U (2007) TRPV1 mediates histamine-induced itching via the activation of phospholipase A2 and 12-lipoxygenase. J Neurosci 27:2331-2337.

Wilson SR, Gerhold KA, Bifolck-Fisher A, Liu Q, Patel KN, Dong X, and Bautista DM (2011) TRPA1 is required for histamine-independent, Mas-related G proteincoupled receptor-mediated itch. Nat Neurosci 14:595-602

Yaksh TL, Ozaki G, McCumber D, Rathbun M, Svensson C, Malkmus S, and Yaksh MC (2001) An automated flinch detecting system for use in the formalin nociceptive bioassay. J Appl Physiol (1985) 90:2386-2402.

Yosipovitch G, Greaves MW, and Schmelz M (2003) Itch. Lancet 361:690-694.

Address correspondence to: Dr. Tony L. Yaksh, Department of Anesthesiology, University of California, San Diego, 9500 Gilman Drive, Mail Code 0818, San Diego, La Jolla, CA 92093-0818. E-mail: tyaksh@ucsd.edu 